\title{
Numerical Calibration of Mass Flow Plug for Inlet Testing
}

\author{
Jonathan Sasson $^{1}$ \\ Case Western Reserve University, Cleveland, OH, 44106 \\ Paul J. Barnhart ${ }^{2}$ \\ Case Western Reserve University, Cleveland, OH, 44106 \\ and \\ David O. Davis ${ }^{3}$ \\ NASA Glenn Research Center, Cleveland, OH, 44135
}

\begin{abstract}
A simple control volume model has been developed to calculate the discharge coefficient through a mass flow plug (MFP) and validated with a calibration experiment. The maximum error of the model within the operating region of the MFP is $0.54 \%$. The control volume analysis developed work is comprised of a sequence of flow calculations through the MFP. The model uses the MFP geometry and operating pressure and temperature to couple continuity, momentum, energy, an equation of state, and wall shear. The discharge coefficient calculation also includes the effects of boundary layer growth, including the reduction in cross-sectional flow area as characterized by the boundary layer displacement thickness. The last calculation in the sequence uses an integral method to calculate the growth of the boundary layer, from which the displacement thickness is then determined. The result of these successive calculations is an accurate one-dimension model of the velocity, pressure, and temperature through the MFP. For comparison, a computational fluid dynamic (CFD) calibration is shown, which when compared to the presented numerical model, had a lower accuracy with a maximum error of $1.35 \%$ in addition to being slower by a factor of 100."
\end{abstract}

\section{Nomenclature}

$\begin{array}{ll}A & =\text { area } \\ A R & =\text { area ratio } \\ C_{D} & =\text { discharge coefficient } \\ C_{f} & =\text { skin friction coefficient } \\ F_{c} & =\text { van Driest's parameter } \\ F_{i} & =\text { algebraic functions in Walz's model } \\ H & =\text { shape factor } \\ L & =\text { length } \\ \dot{m} & =\text { mass flow rate } \\ M & =\text { Mach number } \\ P & =\text { pressure } \\ P r & =\text { Prandtl number } \\ R & =\text { specifc gas constant } \\ \operatorname{Re} & =\text { Reynolds number } \\ S & =\text { surface area } \\ T & =\text { temperature } \\ U & =\text { velocity } \\ W & =\text { shape factor } \\ Z & =\text { momentum thickness parameter } \\ \gamma & =\text { specific heat ratio } \\ \delta & =\text { velocity boundary layer thickness }\end{array}$

\footnotetext{
${ }^{1}$ Student, Mechanical and Aerospace Engineering Dept., AIAA Student Member.

${ }^{2}$ Associate Professor Title, Mechanical and Aerospace Engineering Dept., AIAA Professional Member.

${ }^{3}$ Aerospace Engineer, Inlet and Nozzle Branch, Senior AIAA Member.
}

American Institute of Aeronautics and Astronautics 


$\begin{array}{ll}\delta^{*} & =\text { displacement thickness } \\ \delta_{3} & =\text { dissipation thickness } \\ \mu & =\text { viscosity } \\ \phi & =\text { cone angle } \\ \theta & =\text { momentum thickness } \\ \rho & =\text { density } \\ \tau & =\text { shear stress } \\ \Omega & =\text { square root of } F_{c}\end{array}$

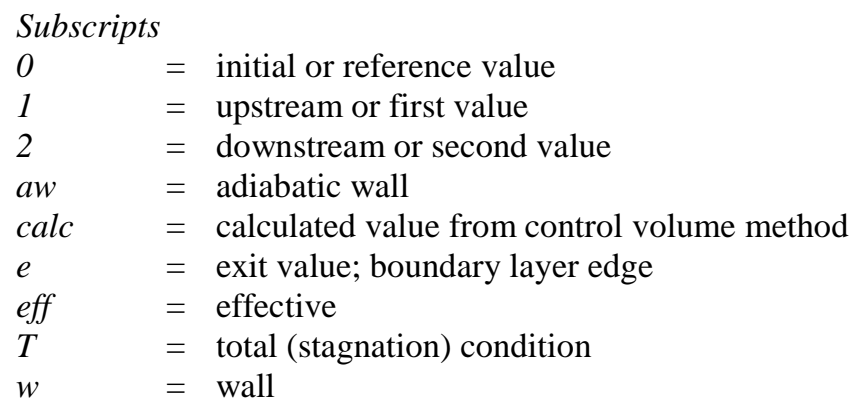

\section{Introduction}

Drior to coupling a high-speed inlet to an actual turbine engine, the performance of the inlet is usually evaluated by testing the inlet with a device that simulates the air flow demand of the engine. The inlet must also hold stable operation over a range of mass flow rates. Accurate measurement of the mass flow rate is therefore necessary. Due to its mechanical simplicity, the air flow through an inlet is often controlled and measured with a Mass Flow Plug (MFP) which is typically a pipe whose exit area is regulated by translating a body of revolution along the axis of the pipe ${ }^{1}$.

The body of revolution is usually conical, but other shapes have been used, and the pipe may either be constant area with a sharp corner or variable area with a contour at the exit. Similar mass flow plugs are also used during the testing phase to control and measure boundary-layer bleed flow typically incorporated in high-speed inlets for shock wave stability and enhanced performance. Figure 1 shows the MFP installed on a Boeing test. This MFP and configuration are used in the analysis.

Due to the high costs of wind tunnel testing, it is often not feasible to calibrate the flow nozzle or the MFP. Recently, there has been a substantial increase in the demand of computational fluid dynamics (CFD) predictions since these are cheaper. They are still time consuming and require the researcher to work closely with a CFD analyst. The goal of this analysis is to create a one-dimensional model that can be implemented by a researcher for any calibration geometry, and obtain a solution that is of comparable accuracy as the CFD results.

A rapid method for calculating the discharge coefficient, $C_{D}$, for a MFP is desired that can be used in lieu of an experimentally determined calibration. The method must be able to capture the geometric variations of typical mass flow plugs operating under a range of flow rates, pressures, and temperatures. A control volume analysis has been developed to perform this calculation of the discharge coefficient for nominal, undistorted, axisymmetric flows through a MFP.

\section{Analysis Methodology}

The control volume analysis developed for this work is comprised of a sequence of flow calculations through the MFP. The first pass uses an inviscid, compressible one-dimensional stream-tube model. The second pass calculates a wall shear based on the results from the first inviscid calculation using the flow properties at the edge of the boundary layer using a compressible, turbulent skin friction model. The third pass solves a coupled set of onedimensional relations for conservation of mass, momentum, energy, an equation of state, and the wall shear calculated in the second calculation. The result of these successive calculations is an accurate one-dimension model of the velocity, pressure, and temperature through the MFP.

The discharge coefficient calculation must also include the effects of boundary layer growth, and the reduction in cross-sectional flow area as characterized by the boundary layer displacement thickness. The last calculation in the 
sequence uses an integral method to calculate the growth of the boundary layer, from which the displacement thickness is then determined.

Figure 2 shows the basic physical elements of the calibration rig. Locations of quantities of interest are also shown in Fig. 2. The flow is assumed choked at the MFP throat, therefore the exit Mach number, $M_{e}$, is 1 . The ideal mass flow rate is determined by the intake total pressure, $P_{T 0}$, the total temperature, $T_{T 0}$, and the MFP minimum area, $A_{e}$. The exit total pressure, $P_{T e}$, is calculated by accounting for losses in the rig. This pressure is used to calculate the actual mass flow rate.

The calibration process is modeled by a series of control volumes across the calibration rig as shown in Fig. 2 . The analysis is also split into four sequential steps. The first step calculates the thermodynamic properties across each control volume isentropically. The second step uses the previous solutions to calculate the wall friction losses. The third step ties together the losses with the isentropic solution using a fully-coupled system of equations to determine the total pressure loss. Finally, the fourth step computes the displacement thickness to correct the effective flow area reduced by boundary layer displacement. The results from these four steps are used to calculate the discharge coefficient, $C_{D}$.

\section{A. Minimum Geometric Area}

The minimum geometric area (or throat area) of the MFP must be determined to start the first pass of the control volume analysis. The sonic line is assumed to be perpendicular to surface, therefore the minimum geometric area is approximated by a curve, $f(x)$, that is both normal to the cone and the cold pipe's exit as shown in Fig. 3 and revolved around the $\mathrm{x}$-axis.

\section{Control Volume Analysis}

In order to determine the internal flow characteristics of the MFP and calibration rig, it is first discretized into a number of segments. The geometry of each section contributes to create a series of one-dimensional control volumes. The inner surfaces create the real boundaries, and the entrance and exit cross-sectional areas of each segment create the imaginary boundaries of the control volume element. The sum of the volume elements create a stream-tube representation of the internal control volume ${ }^{2}$. Each control volume element is further subdivided into smaller sections to create a stream-tube from very small discretized control volume sections. Figure 4 represents a visualization of a one-dimensional discretized control volume element. The figure also shows the stream-tube the control-volumes create. The internal flow solutions of the one-dimensional stream-tube correspond to physical locations on the calibration rig (shown in Fig. 2).

The required geometric information for each one-dimensional control volume element of Fig. 4 consists of the upstream and downstream areas, $A_{l}$ and $A_{2}$ respectively, and the total surface area, $S$. All flow quantities are assumed constant perpendicular to the flow across the volume elements, and are only calculated at the upstream and downstream boundaries. The flow quantities calculated at each imaginary boundary of the control volume are: density, $\rho$, axial velocity, $U$, pressure, $P$, temperature, $T$, total temperature, $T_{T}$, total pressure, $P_{T}$, Mach number, $M$.

\section{B. Inviscid Solution}

The first step in the methodology is to compute the flow quantities presented in the previous section from a statement of conservation of mass for compressible flow

$$
\frac{A_{1} P_{T 1}}{\sqrt{T_{T 1}}} \sqrt{\frac{\gamma}{R}} M_{1}\left[1+\frac{\gamma-1}{2} M_{1}^{2}\right]^{-\frac{\gamma+1}{2(\gamma-1)}}=\frac{A_{2} P_{T 2}}{\sqrt{T_{T 2}}} \sqrt{\frac{\gamma}{R}} M_{2}\left[1+\frac{\gamma-1}{2} M_{2}^{2}\right]^{-\frac{\gamma+1}{2(\gamma-1)}}
$$

The first pass analysis starts at the bellmouth's entrance and marches downstream across each volume element, using Eq. (1) to determine the downstream Mach number from the known upstream Mach number and cross sectional areas. The downstream Mach number then becomes the upstream Mach number for the next volume element marching the solution downstream. The temperatures and pressures are determined from the known Mach numbers and Eqs. (2) and (3) respectively. Velocity and density are computed from Eqs. (4) and (5). Due to the isentropic assumption, the total pressure and temperature are constant across each volume element.

$$
P=P_{T}\left[1+\frac{(\gamma-1)}{2} M^{2}\right]^{-\frac{\gamma}{\gamma-1}}
$$

American Institute of Aeronautics and Astronautics 


$$
\begin{gathered}
T=T_{T}\left[1+\frac{(\gamma-1)}{2} M^{2}\right]^{-1} \\
U=M \sqrt{\gamma R T} \\
\rho=\frac{P}{R T}
\end{gathered}
$$

\section{Skin Friction Model}

The inviscid flow solution determined in the first step is used to calculate the surface shear force using boundary layer theory. The inviscid velocity, density, temperature, and Mach number become the boundary layer edge quantities, denoted by the subscript $e$. The wall shear per unit surface area is given in Eq. (6) as a function of density and velocity at the boundary layer edge, and the skin friction coefficient, $C_{f}$. Since the edge velocity and density are known from the previous inviscid calculation, the only variable left to be determined is the skin friction coefficient.

$$
\tau_{w}=\frac{1}{2} \rho_{e} U_{e}^{2} C_{f}
$$

The flow is assumed turbulent throughout the calculation. Since the pressure gradient is larger across the throat region and it covers a small segment compared to the rest of the rig, the calculation assumes no significant pressure gradient. With these assumptions, a flat plate compressible turbulent skin friction coefficient formulation of White and Christoph is used. This correlation has proven very accurate over the entire practical range of Reynolds numbers, Mach numbers, and wall temperatures. A complete derivation of the method is found in Ref. 3. For adiabatic flow the skin friction coefficient becomes

$$
C_{f} \approx \frac{0.455}{\Omega^{2} \ln ^{2}\left(\frac{0.06}{\Omega} \frac{R e_{L}}{2} \frac{\mu_{e}}{\mu_{w}} \sqrt{\frac{T_{e}}{T_{a w}}}\right)}
$$

$\Omega$ is the square root of the van Driest's parameter $F_{c}$. The details of this parameter are also presented in Ref. 3 .

$$
\Omega=\frac{\sqrt{\frac{T_{a w}}{T_{e}}-1}}{\sin ^{-1} a^{3 / 2}+\sin ^{-1} \frac{1}{2 \sqrt{a}}}
$$

where

$$
a=\sqrt{\frac{\gamma-1}{2} M_{e}^{2}\left(1+\operatorname{Pr}^{1 / 3} \frac{\gamma-1}{2} M_{e}^{2}\right)^{-1}}
$$

The predominant term in the calculation of the skin friction coefficient is the Reynolds number based on length. The length used to calculate the Reynolds number is not a physical length but rather is defined by Eq. (10). It is determined by dimensional arguments. Since the volume element does not account for an axial length, an effective length is approximated by the surface area, $S$, of the volume element. The running length is the sum of all the effective lengths of the preceding volume elements.

$$
L=\frac{1}{\sqrt{\pi}} \sum_{n=2}^{N} \frac{\sqrt{S_{n}^{2}-\left(A_{n}-A_{n-1}\right)^{2}}}{\sqrt{A_{n-1}}+\sqrt{A_{n}}}
$$

\section{Coupled System/Viscous Model}

The results of the second step do not contain the necessary information to calculate the discharge coefficient. The effects of wall shear calculated in the second step violate the assumptions of the isentropic flow model used in the first step. A third step is required to couple the effects of wall shear stress on the internal MFP calibration rig flow solutions. Wall shear stress creates a small loss in total pressure which results in a reduction in mass flow compared to the one calculated by isentropic assumptions in the first step.

The 7 downstream quantities $\left(\rho_{2}, U_{2}, T_{2}, P_{2}, T_{T 2}, P_{T 2}\right.$, and $\left.M_{2}\right)$ are determined from the seven known upstream quantities $\left(\rho_{l}, U_{l}, T_{1}, P_{l}, T_{T l}, P_{T l}\right.$, and $\left.M_{1}\right)$ including the effects of wall shear ${ }^{2}$. Figure 4 is a representation of this system. The 7 necessary governing equations to solve for the 7 unknowns are presented in Eqs. (11) through (17). 
These relations create a system of equations that must be solved simultaneously, and a multi-variable NewtonRaphson iteration scheme is used. Each term in the equation are once again normalized to provide a value close to unity to aid in the convergence of the iteration scheme. The isentropic flow solution is used as the initial guess for the 7 variables in the iteration technique. The 7 equations represent characteristics every physical control volume consists of ${ }^{4}$

1) Equation of state for an ideal gas:

$$
\frac{\rho_{2}}{\rho_{1}} \frac{P_{1}}{P_{2}} \frac{T_{2}}{T_{1}}-1=0
$$

2) Conservation of mass across the volume element:

$$
\frac{\rho_{2}}{\rho_{1}} \frac{U_{2}}{U_{1}} \frac{A_{2}}{A_{1}}-1=0
$$

3) Conservation of momentum across the volume element:

$$
\frac{\rho_{1} U_{1} U_{2} A_{1}-\rho_{1} U_{1}^{2} A_{1}+P_{2} A_{2}-P_{1} A_{1}-\bar{P}\left(A_{2}-A_{1}\right)+\bar{\tau} S}{\left(P_{1}+\frac{1}{2} \rho_{1} U_{1}^{2}\right) A_{1}}=0
$$

4) Conservation of energy across the volume element:

$$
1-\frac{T_{T 2}}{T_{T 1}} \frac{\rho_{2}}{\rho_{1}} \frac{U_{2}}{U_{1}} \frac{A_{2}}{A_{1}}=0
$$

5) Relation between total and static temperature for an ideal gas:

$$
\frac{T_{T 2}}{T_{T 1}}-\frac{T_{2}}{T_{1}}\left[\frac{1+\frac{\gamma-1}{2} M_{2}^{2}}{1+\frac{\gamma-1}{2} M_{1}^{2}}\right]=0
$$

6) Relation between total and static pressure for an ideal gas:

$$
\frac{P_{T 2}}{P_{T 1}}-\frac{P_{2}}{P_{1}}\left[\frac{1+\frac{\gamma-1}{2} M_{2}^{2}}{1+\frac{\gamma-1}{2} M_{1}^{2}}\right]^{\frac{\gamma}{\gamma-1}}=0
$$

7) Relation between velocity and Mach number for an ideal gas:

$$
\frac{U_{2}}{U_{1}} \frac{M_{1}}{M_{2}} \sqrt{\frac{T_{1}}{T_{2}}}-1=0
$$

The third step in the analysis is similar to the first step in that it computes all the flow properties marching through the volume elements to determine the internal flow. The difference here is that the total pressure does not remain constant because of the wall shear stress effects. Even though total temperature remains constant since there is no heat addition, it is treated as an unknown in the system of equations for completion. It also serves as a redundancy in the calculations. If the total temperature changes, then there is an error in the solution.

\section{E. Boundary Layer Analysis}

The third pass analysis determined the internal flow properties for the calibration rig, however this is still insufficient information to accurately determine the discharge coefficient. Although the previous calculations took into consideration friction losses on total pressure effects, they did not account for the boundary layer displacement thickness which reduces the effective flow area. The third pass determined mass flow rate is lower than the first pass inviscid mass flow rate, but it yields an unrealistic discharge coefficient around 0.99. A fourth step is therefore necessary to determine the boundary layer growth and the displacement thickness. A von Karman momentum integral equations is applied in this step.

American Institute of Aeronautics and Astronautics 
The simple momentum integral equation presented in Eq. (18) represents a reliable method for calculating the boundary layer displacement thickness. The equation applies to both laminar and turbulent flows. For compressible flow it is necessary to include Mach number effects ${ }^{5}$.

$$
\frac{d \theta}{d x}+\frac{\theta}{U_{e}}\left(2+H-M_{e}^{2}\right) \frac{d U_{e}}{d x}=\frac{1}{2} C_{f}
$$

Equation (18) has too many unknowns $(\theta, H)$. The skin friction coefficient is defined by Eq. (7). Walz's method is applicable to both laminar and turbulent boundary layer methods in both low- and high-speed flows ${ }^{3}$. The method introduces a momentum thickness parameter, $Z$, and another shape factor, $W$.

$$
\begin{gathered}
Z=\theta\left(\frac{\rho_{e} U_{e} \theta}{\mu_{w}}\right)^{n} \\
W=\frac{\delta_{3}}{\theta}
\end{gathered}
$$

where $n=1$ for laminar flow and $n=0.268$ for turbulent flow, and $\delta_{3}$ is the compressible kinetic energy thickness. The two coupled first order differential Eqs. (20) and (21) are obtained by the substitution of $Z$ and $W$ in the momentum and mechanical energy integral relations. Only the parameters $Z$ and $W$ are necessary in these equations

$$
\begin{gathered}
\frac{d Z}{d x}+\frac{F_{1}}{U_{e}} \frac{d U_{e}}{d x} Z=F_{2} \\
\frac{d W}{d x}=\frac{F_{3}}{U_{e}} \frac{d U_{e}}{d x} W=\frac{F_{4}}{Z}
\end{gathered}
$$

The four functions $F_{i}$ are algebraic functions of the Mach number, Reynolds number based on momentum thickness $\left(\operatorname{Re}_{\theta}=\frac{\rho_{e} U_{e} \theta}{\mu_{w}}\right)$, and shape factor $H=\delta^{*} / \theta$. The formulas are shown in Ref. 3 .

The two differential Eqs. (20) and (21) are integrated using a 4th Order Runge-Kutta integration method to solve for $\mathrm{Z}$, and $\mathrm{W}^{6}$. The integration is carried downstream starting from bellmouth entrance. The integration is marched downstream from the starting point across volume elements. The initial conditions at the upstream location of the control volume are used to solve for the downstream values of $Z, W$, and $\theta$. The downstream values then become the upstream values, and thus the initial conditions for the next volume element.

Once the downstream values of $Z, W$, and $\theta$. are determined for the last volume element, $\delta^{*}$ is calculated. The MFP's throat radius is reduced using $\delta^{*}$, from which an effective throat area is calculated. The ideal mass flow rate through the calibration rig is determined by using the MFP's throat area, and the supply total pressure and temperature. Since the flow is choked at this point the flow rate is given by

$$
\dot{m}_{\text {ideal }}=\frac{A^{*} P_{T}}{\sqrt{T_{T}}} \sqrt{\frac{\gamma}{R}}\left[\frac{\gamma+1}{2}\right]^{-\frac{\gamma+1}{2(\gamma-1)}}
$$

The viscous mass flow rate is finally computed from Eq. (23) by correcting the calculated mass flow rate from the third pass by the ratio of effective flow area to throat area (minimum geometric area). Finally, the discharge coefficient is determined from Eq. (24).

$$
\dot{m}_{\text {actual }}=\dot{m}_{\text {calc }} \frac{A_{\text {eff }}}{A^{*}}
$$

where $\dot{m}_{\text {calc }}$ is the mass flow rate calculated in the third pass.

$$
C_{D}=\frac{\dot{m}_{\text {actual }}}{\dot{m}_{\text {ideal }}}
$$

The analysis is presented in greater detail in Ref. 7.

\section{Experimental Setup and Procedure}

The test article assembly is shown in Fig. 5. The N+2 MFP and the contraction assembly were designed and fabricated by Boeing. The remainder of the components were designed and fabricated specifically for this test. Ambient air is drawn through the ASME nozzle which serves as the primary reference mass-flow measurement device.

American Institute of Aeronautics and Astronautics 
In this experiment the flow from the ASME nozzle exhausts directly into a settling chamber which acts as a plenum. The intent of the plenum chamber was for the flow through the mixing pipe to settle. The nozzle is directly connected to the settling chamber via an interface flange. An O-ring sitting in a groove on the nozzle's flange creates a seal on the interface flange to prevent and flow leakage. From the settling chamber, the flow passes through a 22:1 contraction and enters the MFP. The plenum attaches directly to the bellmouth. The MFP assembly is held in place via a support structure specifically designed for this experiment.

The MFP assembly consists of a diffuser with an Aerodynamic Interface Plane (AIP) pressure rake at its 1.5 diameter entrance that expands to 1.994 inches. It also has a cold pipe of the same diameter with an MFP pressure rake and a contoured exit. The MFP rake is 2 inches from the pipe exit. The 2 inch mass flow plug sits at various positions inside the pipe. The entire test article assembly is mounted on top of the wind tunnel test section as shown in Fig. 5. After passing through the MFP, the flow exhausts into the test section which is maintained at approximately 1.75 psia (altitude exhaust). The resultant pressure ratio is 8.22 ensuring a choked MFP.

\section{A. AIP Station}

The AIP rake sits at the entrance of the 1.5 inch diameter diffuser. The rake follows the guidelines established in ARP 1420 to measure total pressure at the AIP $^{8}$. It represents an engine face plane which is defined by the leading edge of the most upstream engine strut, vane, or blade row. The station contains eight equally-spaced rakes with five pitot tubes per rake for a total of 40 total pressures. The pitot tubes are all area-weighted, i.e. the area covered by each ring is the same. Figure 6 represents the layout of the rake. The figure shows an upstream view of the rake; it represents the pressure distribution a compressor "sees". The nomenclature used in Fig. 6 that refers to the individual pitot tubes $(i, j)$ is per ARP $246^{9}$. The ring number is defined by $i$, and the rake number by $j$.

\section{B. MFP Station}

The MFP station records the conditions immediately upstream of the mass flow plug. This station contains four equally-spaced rakes with three pitot tubes per rake for a total of 12 total pressures. These are clocked 22.5 degrees counter clockwise (looking upstream) as to avoid the wakes of the upstream AIP rakes. The tubes on these of rakes are also positioned radially so as to have equal area weighting. There are no wall static taps at the MFP station. The layout of the rake is shown in Fig. 7 in an upstream view.

\section{ASME Nozzle}

The ASME Long-Radius Flow Nozzle is one the most important components of the testing section as it serves two purposes. It draws the fluid into the settling chamber, and measures the actual mass flow rate flowing through the MFP. The nozzle generates differential pressure which is used to determine the mass flow rate. The flow rate is corrected by a nozzle discharge coefficient.

With the geometry known, the air flowing through the nozzle can be approximated as an ideal gas. The wall taps measure the differential pressure and determine the static pressure at the throat. They are shown in the overall system description of Fig. 5. Using the atmospheric pressure as the total pressure and the measured static pressure, the throat Mach number is calculated from Eq. (25).

$$
M_{A S M E}=\sqrt{\frac{2}{\gamma-1}\left({\frac{P_{T 0}}{P}}^{\frac{\gamma-1}{\gamma}}-1\right)}
$$

The final parameter needed to calculate the flow rate is the nozzle discharge coefficient. It takes into account any loses due to viscous effects, and permanent head loss. The nozzle is calibrated in the flow lab at GRC to determine a correlation for discharge coefficient based on the throat Reynolds number. The calibration curve and the Reynolds number calculation are presented in Section V. Finally, the mass flow rate is determined from the Mach number and discharge coefficient where $A_{A S M E}$ is the throat cross-sectional geometric area.

$$
\dot{m}=C_{D} A_{A S M E} \frac{P_{T 0}}{\sqrt{T_{T 0}}} \sqrt{\frac{\gamma}{R}} M_{A S M E}\left[1+\frac{\gamma-1}{2} M_{A S M E}^{2}\right]^{-\frac{\gamma+1}{2(\gamma-1)}}
$$

\section{Error Analysis}

A general uncertainty analysis was performed for the calibration. The uncertainty in the discharge coefficient is estimated by

American Institute of Aeronautics and Astronautics 


$$
\delta C_{D}=\sqrt{\sum_{i=1}^{N}\left(\frac{\partial C_{D}}{\partial x_{i}} \delta x_{i}\right)^{2}}
$$

where $x_{i}$ are the measured quantities and are summarized in Table 1.

\begin{tabular}{lc}
\hline \multicolumn{1}{c}{ Source } & Value \\
\hline ASME throat diameter & 0.0005 in \\
ESP pressure reading & $0.0029 \mathrm{in}$ \\
Differential pressure on ASME nozzle & $0.0050 \mathrm{psi}$ \\
Thermocouples & $0.0010 \mathrm{R}$ \\
Plug position & $0.0010 \mathrm{in}$ \\
\hline
\end{tabular}

Table 1. Summary of uncertainties

\section{E. Baseline Calibration Procedure}

The calibration begins by positioning the plug $95 \%$ of the way forward from the jackscrew housing. The plug is not closed all the way to avoid damaging the MFP and the cold pipe. The back pressure is then dropped to approximately 1.75 psia. The plug is opened by increments of $5 \%$ of the closed plug position to measure the mass flow rate at various plug positions. The Mach number at the AIP is monitored during testing and the plug is opened until the Mach number is approximately 0.7 to simulate realistic engine operation. At each plug position the pressure is first allowed to settle for a few seconds before a data sample is recorded. Each sampling records the data over 10 seconds and averages the data to obtain a single reading for each instrument.

\section{Results}

The experiment was performed five times without any distortion devices to produce a baseline calibration using non-distorted flow. The results of this calibration are presented in Fig. 8 showing the MFP's discharge coefficient, $C_{D}$, and the MFP area ratio, $A R$. The area ratio is defined as the throat area determined previously over the cold pipe's maximum cross-sectional area.

$$
A R=\frac{A^{*}}{A_{\max }}
$$

The experimental results are compared against predictions made by the control volume method presented in previously and a CFD calibration. The details of the CFD calibration are presented in Ref. 10. The ideal mass flow rate is calculated from the total upstream conditions and the throat area for all solutions. The region of interest in the calibration lies between an area ratio of 0.3 and 0.5 . It is in this region that the MFP is used as a flow meter during wind tunnel testing. In this region the maximum deviation of the control volume calculations from the experimental results is $0: 54 \%$. The maximum deviation in the CFD results is $1.35 \%$. The maximum experimental error in the operating range is $0.57 \%$. The locations of these errors are shown in Fig. 8 by the small arrows. The control volume method is within the error bounds of the experimental data. Both the control volume method and the CFD analysis are within the experimental error in the operating range.

The simple control volume analysis developed from first principals has a higher accuracy than the CFD solution calibration by approximately $0.8 \%$. Figure 8 shows the control volume method is a reliable alternative to calibrate a MFP assembly. It also reduces the pre-computational time since no grid is required, only a simple description of the physical geometry. Each CFD solution took about 8 hours to converge using the supercomputer clusters at NASA Ames, while the control volume method took 5 minutes to calculate the calibration curve over the range of MFP area ratios presented.

The actual experimental flow rate is calculated from the ASME nozzle. The nozzle's discharge coefficient is determined from a metrology calibration. The calibration is presented in Fig. 9 showing the nozzle's discharge coefficient and the Reynolds number at the throat, $R_{A S M E}$.

The metrology results show a significant amount of scatter, with a maximum deviation of $1.52 \%$ at $R e_{A S M E}=$ 100,000 , and do not cover the entire range tested during the MFP calibration. The discharge coefficient has a sudden 
drop for Reynolds numbers lower than 50,000, however no test data is taken below 65,000. A least squares fit is used to determine the discharge coefficient between the minimum and the maximum tested Reynolds number. This curves matches the typical curve of an ASME nozzle drawing air from the atmosphere as shown by the dashed curve in Fig. 9. The typical wind tunnel test operating range lies in the region calibrated in the metrology lab. The scatter could account for the increased error in the control volume method outside the operating range in Fig. 8 . The maximum error in the control volume method is $1.79 \%$. This maximum error is near the plug's minimum limit.

Wind tunnel testing engineers at NASA use the total pressure measured by the MFP rakes instead of the total upstream pressure to calculate a reduced "ideal" flow rate to calculate the discharge coefficient. Therefore this discharge coefficient does not account for any viscous losses upstream of the plug. The results of calculating the discharge coefficient with this reduced ideal mass flow rate are presented in Fig. 10. The figure also includes calibration results performed by Boeing who use the MFP pressures to correct their calibration. The behavior of the curves shown in Fig. 10 is vastly different than in Fig. 8. Viscous effects are not prevalent in the operating range, which is not the expected behavior with increasing mass flow rate. Boeing's calibration is within the experimental error of the new calibration.

\section{Conclusion}

The experimental efforts use a mass flow plug previously employed by Boeing to determine mass flow rate through a low sonic boom inlet. The rig consists of a small diffuser, a straight length segment with a contoured exit for the flow to settle and a plug. The flow through the MFP is measured with an ASME nozzle calibrated by the metrology lab at the NASA Glenn Research Center. The ASME nozzle calibration was inconsistent at low Reynolds numbers which can be attributed to the larger error in the control volume method outside the operating range.

A simple analytical method of calculating the discharge coefficient, $C_{D}$, for undistorted, axisymmetric flows through a MFP is developed from first principles and verified against experimental results. The control volume method is in excellent agreement with the test data in the typical operating range (with a maximum error of $0.54 \%$ ) of the MFP. The method allows for rapid geometric variations of typical mass flow plugs operating under a range of flow rates, pressures and temperatures. It also accurately couples continuity, momentum, energy, an equation of state, and wall shear. The effects of boundary layer growth and the reduction in cross-sectional flow area (characterized by the boundary layer displacement thickness) are calculated using an integral method. The control volume method is of comparable accuracy to the CFD when compared to the experimental results. The maximum error in the CFD is $1.35 \%$. The main advantage of the control volume method is the rapid setup and computational time.

The CFD results are not capable of predicting the pressure decay through the MFP, with a maximum error of 90\%. The CFD models are run on the supercomputer cluster at NASA Ames using parallel processing. The control volume requires minimal geometric modeling since no grid is required. Computational time is also reduced by a factor of 100 for each plug position. The code may be used by experimentalists to accurately calibrate mass flow plugs and other geometries under choked conditions. The control volume method developed is more reliable and easier to use than CFD. It is recommended as a tool to use before developing calibration experiments.

\section{Figures}

American Institute of Aeronautics and Astronautics 


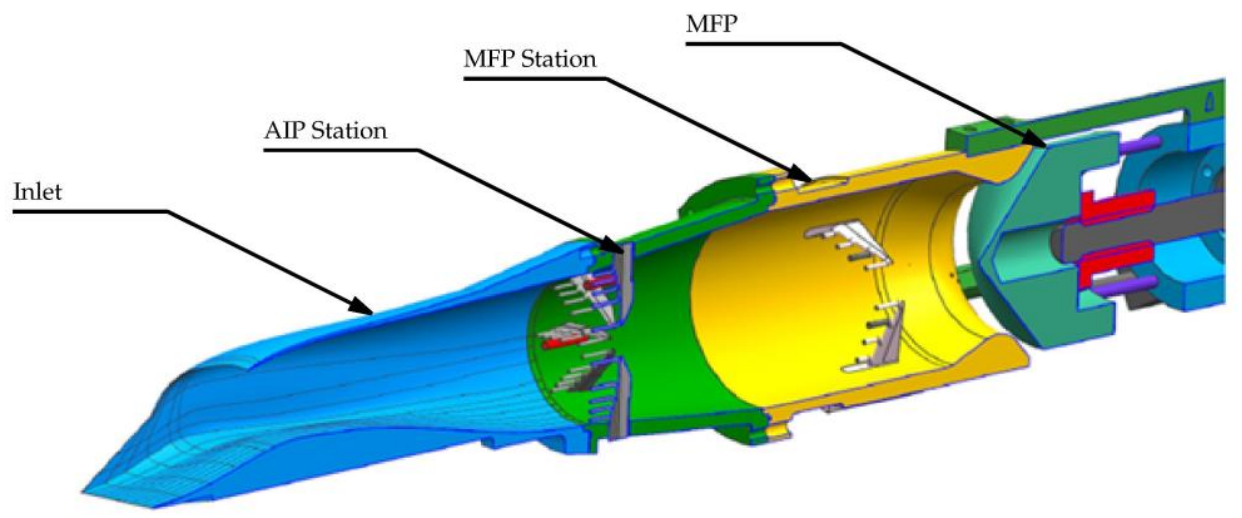

Figure 1. Low-boom inlet/MFP configuration.
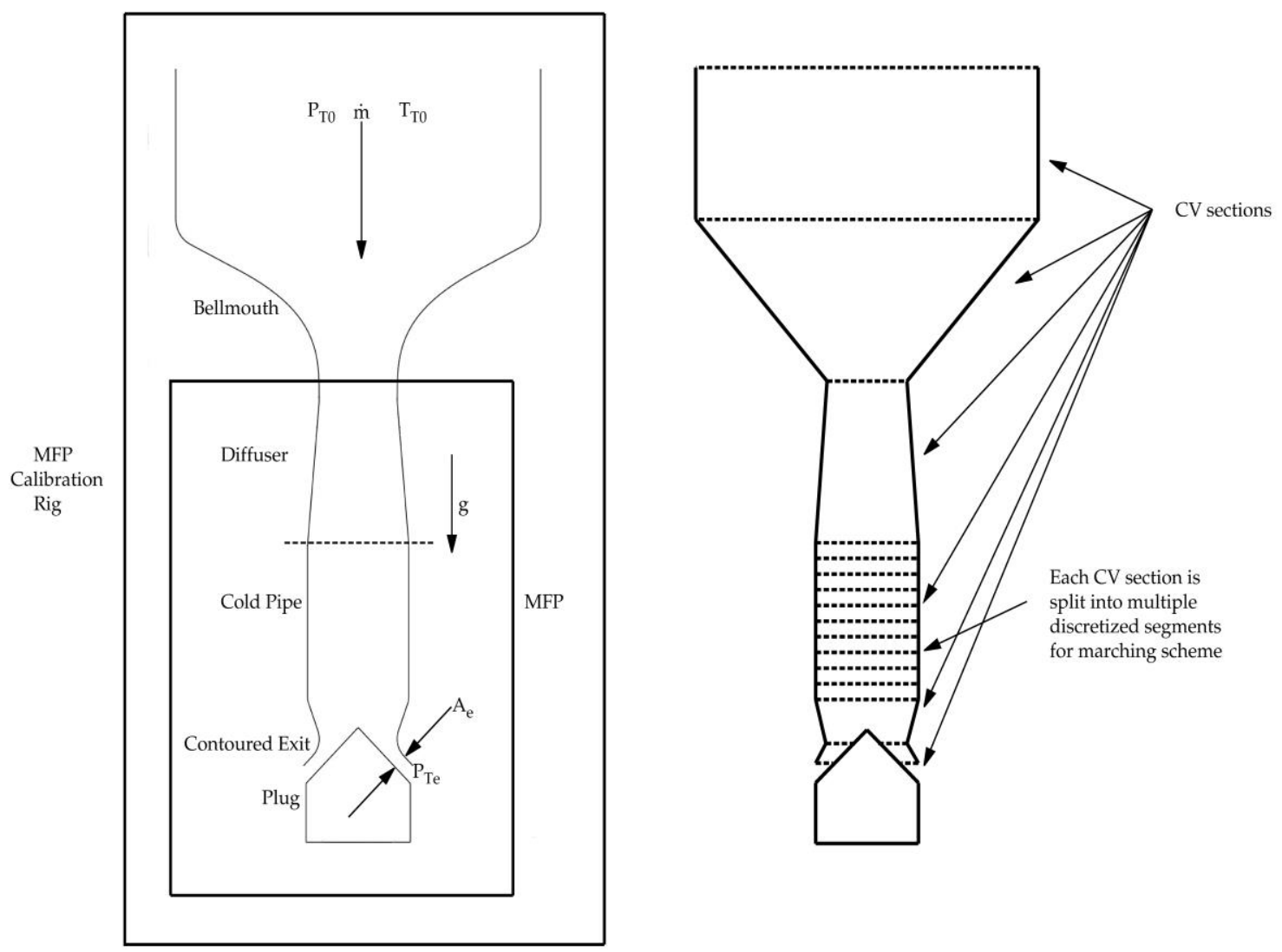

Figure 2. Components of the calibration rig and control volume elements. 


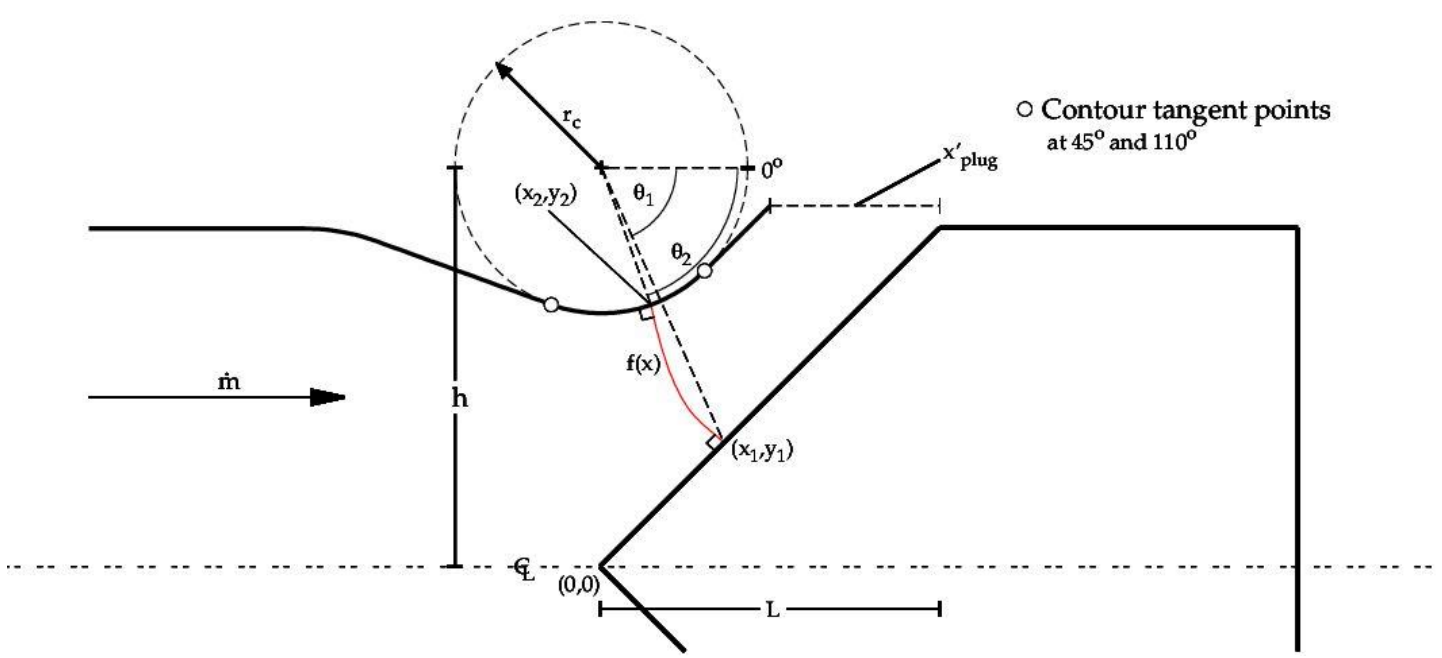

Figure 3. Schematic for calculating MFP minimum area.

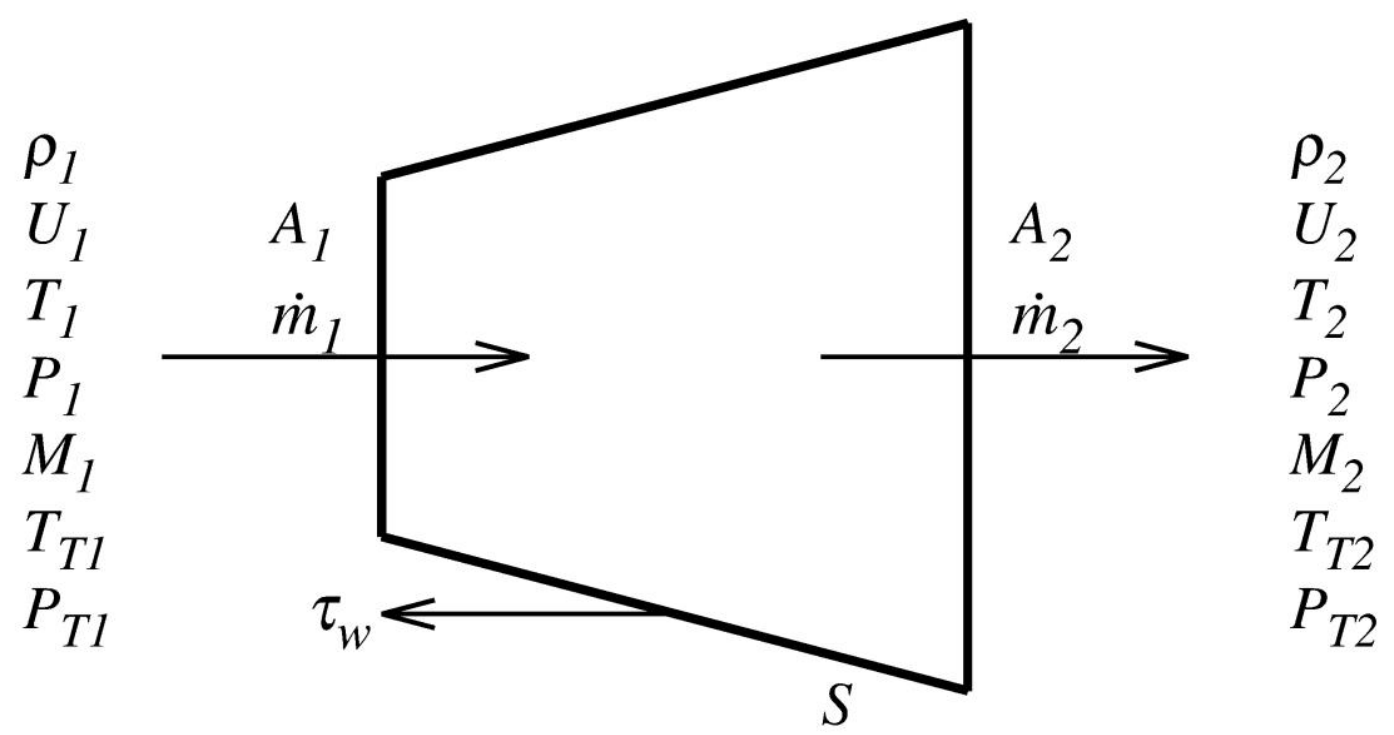

Figure 4. Sample control volume with the flow quantities. 


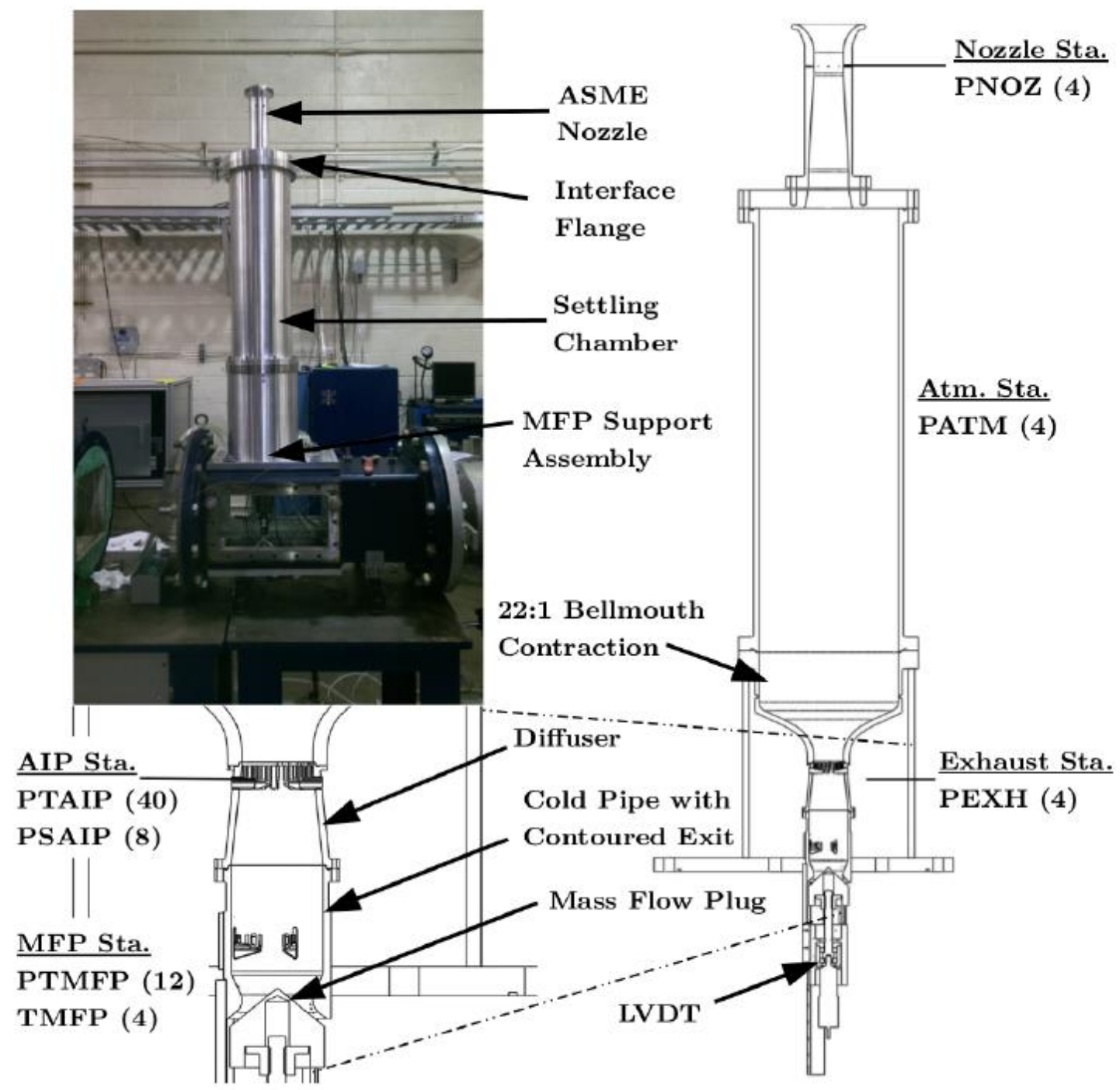

Figure 5. Test setup and instrumentation stations. 


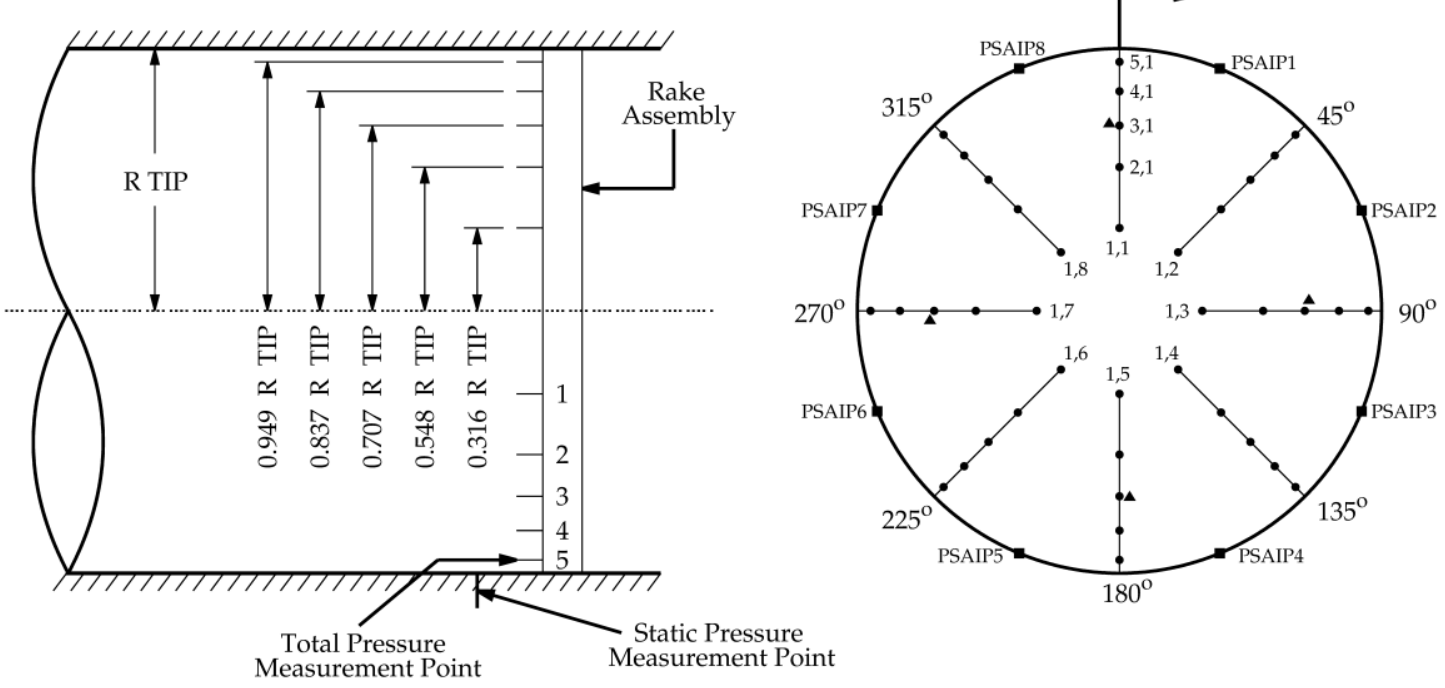

Figure 6. Side view and upstream view of the AIP instrumentation rake.
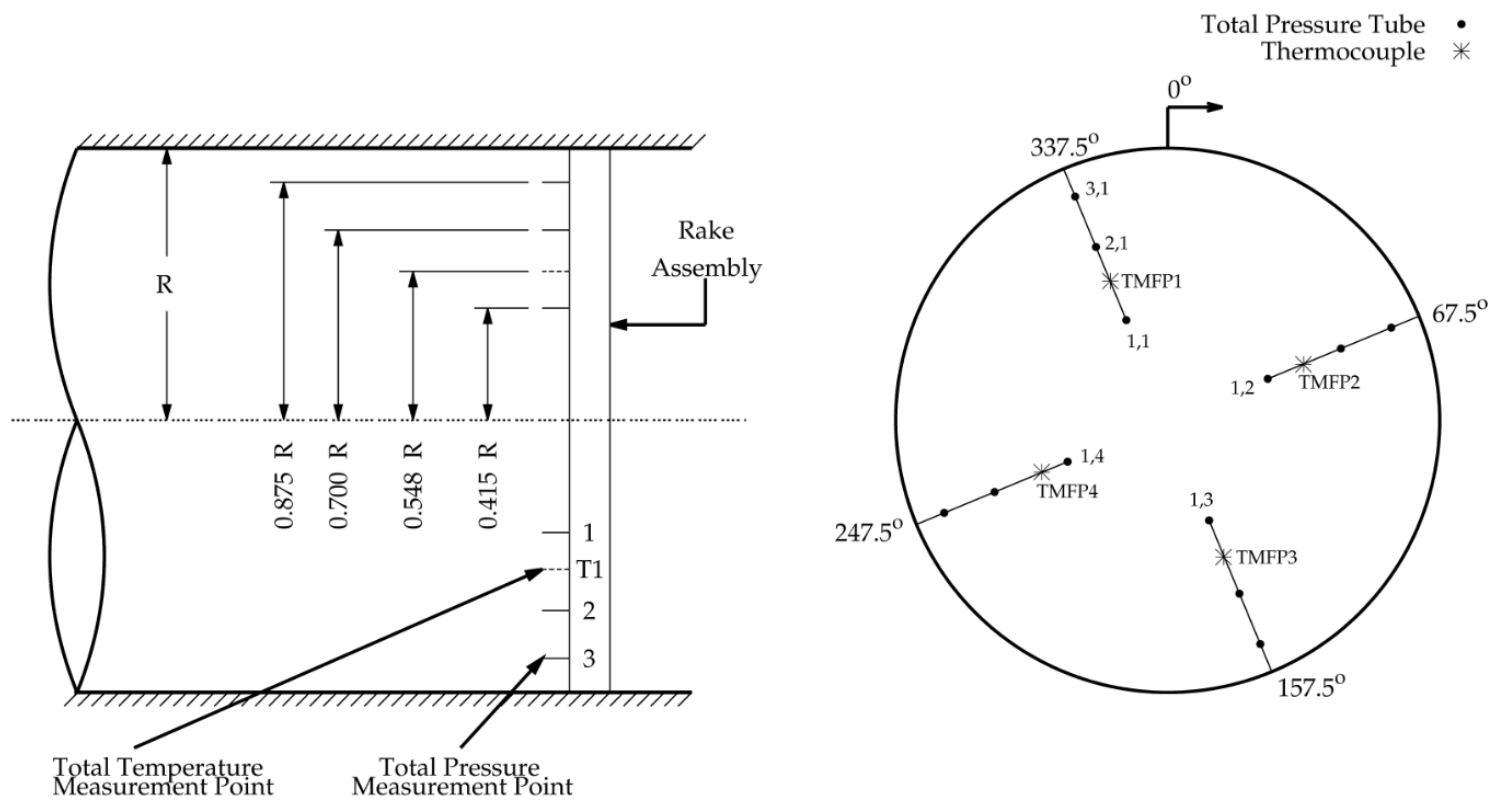

Figure 7. Side view and upstream view of the MFP instrumentation rake 


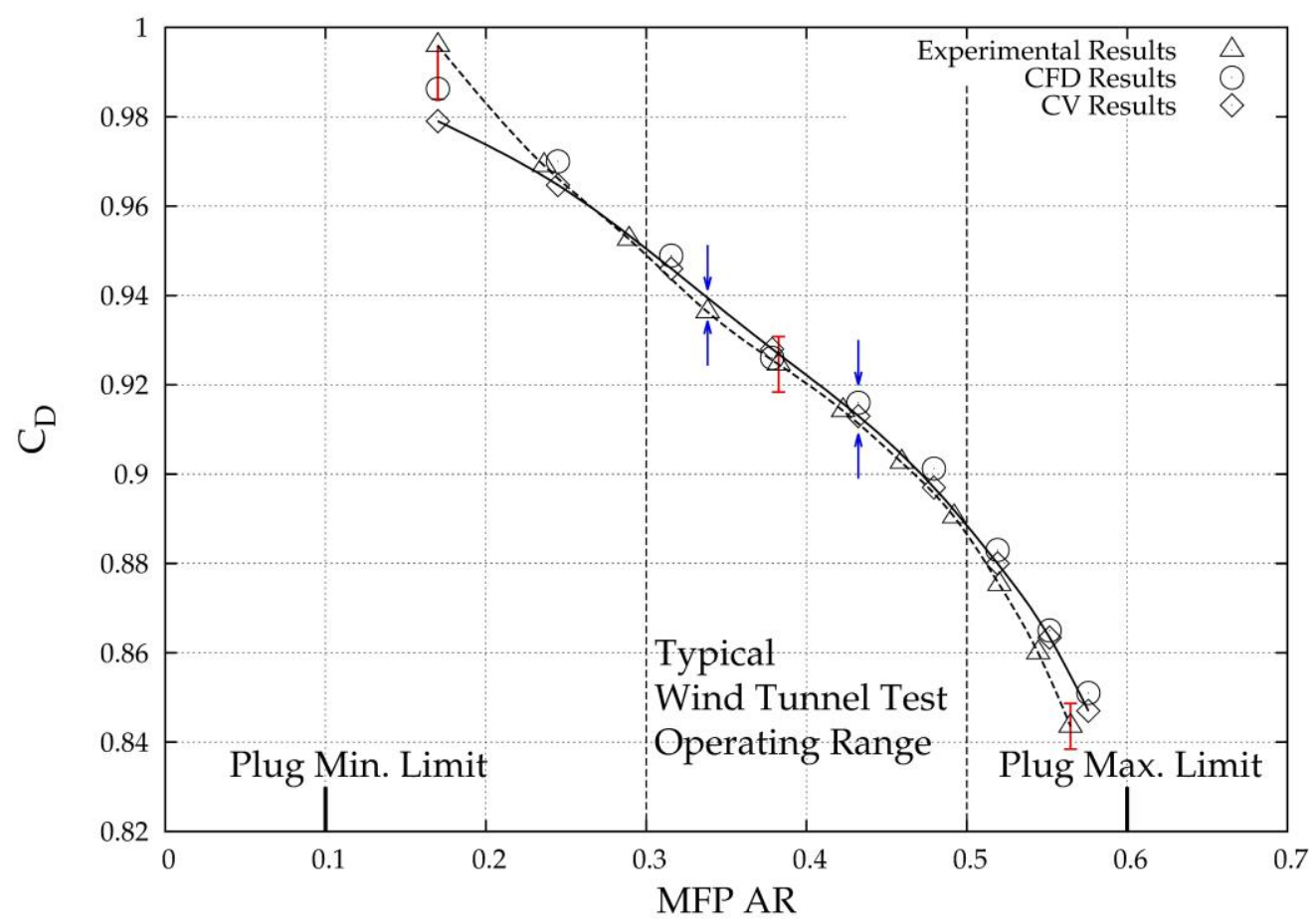

Figure 8. MFP discharge coefficient results

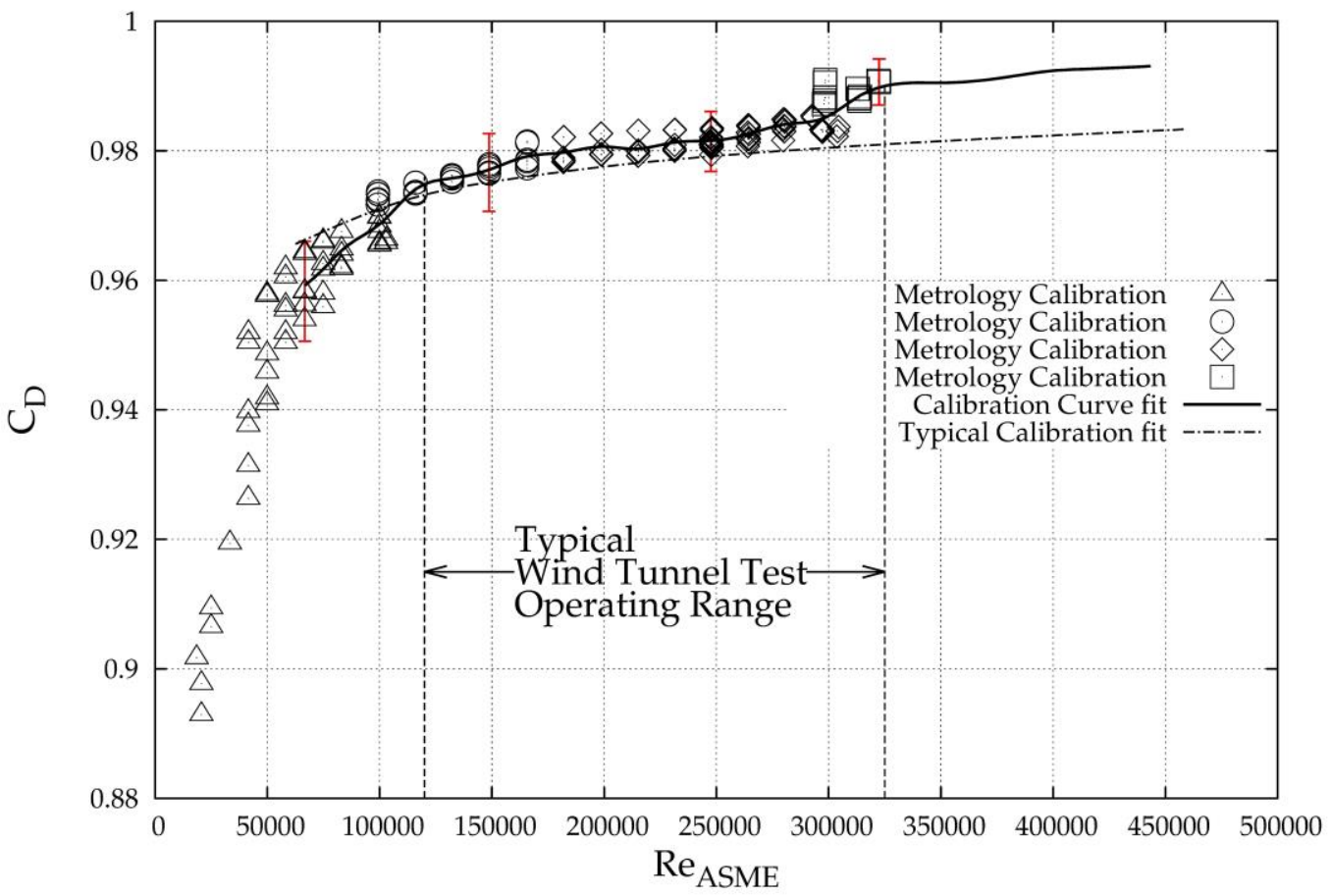

Figure 9. ASME nozzle calibration. 


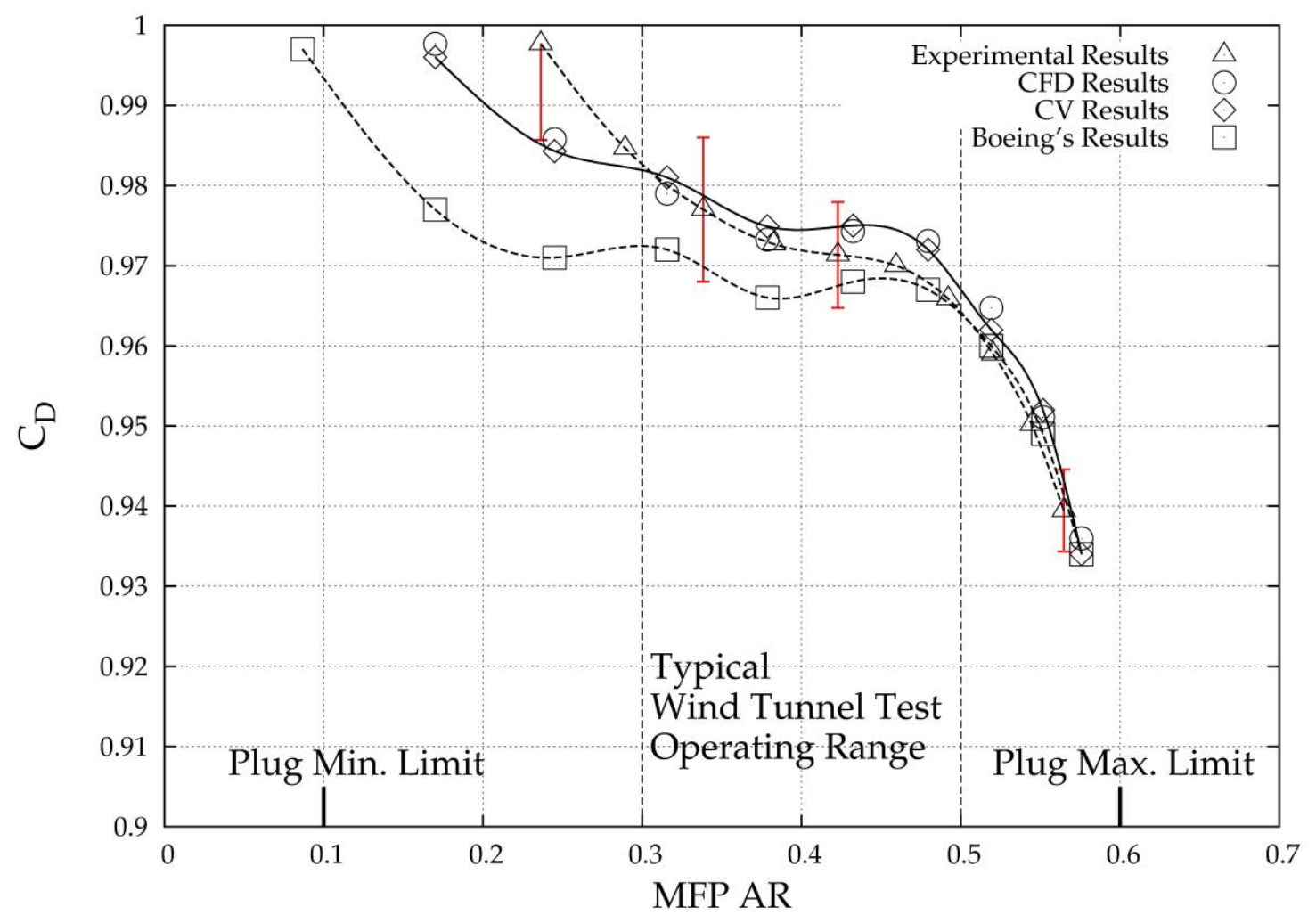

Figure 10. MFP discharge coefficient results with reduced "ideal" mass flow rate.

\section{Acknowledgments}

Funding from the Supersonics Project of the NASA Fundamental Aeronautics Program is gratefully acknowledged.

\section{References}

${ }^{1}$ Davis, D. O., and Saunders J. D., "Calibration of the NASA GRC 16" Mass-Flow Plug," ASME, Rio Grande, Puerto Rico, 2012.

${ }^{2}$ Barnhart, P. J., "NPAC - Nozzle Performance Analysis Code,” NASA CR-204129, 1997.

${ }^{3}$ White, F. M., Viscous Fluid Flow, $3^{\text {rd }}$ ed., McGraw-Hill, New York, 2006, Chaps. 4, 6.

${ }^{4}$ Shapiro, A. H., The Dynamics and Thermodynamics of Compressible Fluid Flow, $2^{\text {nd }}$ ed., John Wiley \& Sons, New York, 1953, Chaps. 6, 8.

${ }^{5}$ Schlichting, H., and Gersten K., Boundary Layer Theory, $8^{\text {th }}$ ed., McGraw Hill, Germany, 2003.

${ }^{6}$ Moran, J., An Introduction to Theoretical and Computational Aerodynamics, Dover Publications, New York, 2003.

${ }^{7}$ Sasson, J., "Small Scale Mass Flow Plug Calibration," Master Thesis, Mechanical and Aerospace Engineering Dept., Case Western Reserve University, Cleveland, OH, 2015.

8"ARP 1420", SAE, Warrendale, PA, 2002.

9"ARP 246", SAE, Warrendale, PA, 2012.

${ }^{10}$ Sasson, J.,"Effects of Distortion on Mass Flow Plug Calibration”, AIAA Propulsion and Energy, 2015 (to be published). 